PROCEEDINGS OF THE

AMERICAN MATHEMATICAL SOCIETY

Volume 132, Number 8, Pages 2321-2326

S 0002-9939(04)07391-5

Article electronically published on February 12, 2004

\title{
THE NUMERICAL RANGE OF A NILPOTENT OPERATOR ON A HILBERT SPACE
}

\author{
MUBARIZ T. KARAEV
}

(Communicated by Joseph A. Ball)

\begin{abstract}
We prove that the numerical range $W(N)$ of an arbitrary nilpotent operator $N$ on a complex Hilbert space $H$ is a circle (open or closed) with center at 0 and radius not exceeding $\|N\| \cos \frac{\pi}{n+1}$, where $n$ is the power of nilpotency of $N$.
\end{abstract}

The purpose of this note is to describe the numerical range of a nilpotent operator on a Hilbert space.

Let $H$ be a complex Hilbert space, with inner product $\langle$,$\rangle , and denote by B(H)$ the algebra of bounded linear operators on $H$. The numerical range $W(T)$ of an operator $T \in B(H)$ is defined by

$$
W(T)=\left\{\langle T x, x\rangle: x \in(H)_{1}\right\},
$$

where $(H)_{1}=\{x \in H:\|x\|=1\}$ is the unit sphere in $H$. The numerical radius $w(T)$ of an operator $T \in B(H)$ is given by

$$
w(T)=\sup \{|\lambda|: \lambda \in W(T)\} .
$$

Haagerup and Harpe $[\mathrm{HH}]$ proved the following sharp estimate for the numerical radius of a nilpotent operator $N \in B(H)$ :

$$
w(N) \leq\|N\| \cos \frac{\pi}{n+1},
$$

where $n$ is the power of nilpotency of $N$. By the Toeplitz-Hausdorff theorem (see [H, GR]) the numerical range $W(N)$ is convex. Then, to prove that $W(N)$ is a disk centered at zero, it suffices to show its circularity, i.e., that each point $\lambda$ is contained in $W(N)$ with the whole circle $|z|=|\lambda|$, which is essentially contained in the paper $[\mathrm{LT}$ by $\mathrm{Li}$ and Tsing.

The present paper presents a new proof of this known result; namely, we give a different proof of circularity of $W(T)$ using the Sz.-Nagy-Foiaş model.

The main result of the paper is

Theorem. Let $H$ be a complex Hilbert space and let $N \in B(H)$ be a nilpotent operator with power of nilpotency $n$. Then the numerical range $W(N)$ of the operator $N$ is a circle (open or closed) with center at 0 and radius not exceeding $\|N\| \cos \frac{\pi}{n+1}$.

Received by the editors December 22, 2002 and, in revised form, April 28, 2003.

2000 Mathematics Subject Classification. Primary 47A12; Secondary 15A45, $42 \mathrm{~A} 05$.

Key words and phrases. Numerical range, numerical radius, nilpotent operator, model operator. 
As noticed already, by the Toeplitz-Hausdorff theorem and by the inequality (1) of Haagerup and Harpe, it remains to establish circularity of $W(N)$, which will be done using the Sz.-Nagy-Foiaş model.

Let us recall that the characteristic function $\Theta_{T}$ of the contraction $T \in B(H)$ is defined by

$$
\Theta_{T}(\lambda)=\left[-T+\sum_{m=1}^{\infty} \lambda^{m} D_{T^{*}}\left(T^{*}\right)^{m-1} D_{T}\right] \mid \mathcal{D}_{T} \quad(\lambda \in \mathbb{D}),
$$

where

$$
\begin{array}{ll}
D_{T}=\left(I-T^{*} T\right)^{\frac{1}{2}}, & D_{T^{*}}=\left(I-T T^{*}\right)^{\frac{1}{2}}, \\
\mathcal{D}_{T}=\overline{D_{T} H}, & \mathcal{D}_{T^{*}}=\overline{D_{T^{*}} H},
\end{array}
$$

and the series is norm convergent.

Let $E_{1}$ and $E_{2}$ be Hilbert spaces, $B\left(E_{1}, E_{2}\right)$ the space of bounded linear operators from $E_{1}$ to $E_{2}$, and $H^{\infty}\left(B\left(E_{1}, E_{2}\right)\right)$ the space of bounded analytic functions on the unit disk $\mathbb{D}=\{z \in \mathbb{C}:|z|<1\}$ taking values in $B\left(E_{1}, E_{2}\right)$. We call a function $\Theta \in H^{\infty}\left(B\left(E_{1}, E_{2}\right)\right)$ inner if $\Theta(\zeta)^{*} \Theta(\zeta)=I_{E_{1}}$ for almost all $\zeta \in \mathbb{T}=\partial \mathbb{D}$, and $*$-inner if $\Theta(\zeta) \Theta(\zeta)^{*}=I_{E_{2}}$ for almost all $\zeta \in \mathbb{T}$. A function that is inner and $*$-inner simultaneously is called a two-sided inner function, where $I_{E_{i}}$ is an identity operator in $E_{i}, i=1,2$. It is known that $\Theta_{T} \in H^{\infty}\left(B\left(\mathcal{D}_{T}, \mathcal{D}_{T^{*}}\right)\right)$.

Recall that a contraction $T \in B(H)$ is said $[\mathrm{SF}]$ to be of class $C_{0}$ if $T$ is completely nonunitary and there is a nonzero function $f$ in $H^{\infty}$ such that $f(T)=0$. Then there is a unique (up to a constant factor of modulus one) nonconstant inner function $u$, called the minimal function of $T$, such that $u(T)=0$. It is a well-known result of Sz.-Nagy and Foias SF that each $T \in C_{0}$ is unitary equivalent to its model operator $M_{\Theta}=P_{\Theta} S_{E} \mid \mathcal{K}_{\Theta}$, acting on the model space $\mathcal{K}_{\Theta}=H^{2}(E) \Theta \Theta H^{2}(E)$. Here $\Theta=\Theta_{T} \in H^{\infty}(B(E))$ is the characteristic function of the contraction $T$ (a two-sided inner function); $E$ is a Hilbert space with $\operatorname{dim} E=\operatorname{dim}\left(I-T^{*} T\right) H ; H^{2}(E)$ is the Hardy space of $E$-valued functions consisting of all Taylor series $f(z)=\sum_{n=0}^{\infty} \hat{f}(n) z^{n}, z \in \mathbb{D}$, where $\hat{f}(n) \in E, n \geq$ $0, \sum_{n=0}^{\infty}\|\hat{f}(n)\|_{E}^{2}<+\infty ; P_{\Theta}=I-\Theta P_{+} \Theta^{*}$ is the orthogonal projection of $H^{2}(E)$ onto $\mathcal{K}_{\Theta}$, where $P_{+}$is the Riesz projector of $L^{2}(E)$ onto $H^{2}(E)$, and $S_{E} f=z f$ is the shift operator on $H^{2}(E)$.

If $T \in C_{0}$ and $u \in H^{\infty}$ is its minimal function, then by the theorem on scalar multiples (see, e.g., $[\mathrm{SF}, \mathbf{N}]$ ) there exists $\Omega \in H^{\infty}(B(E))$ such that

$$
\Theta_{T} \Omega=\Omega \Theta_{T}=u I_{E} .
$$

In particular, if $T$ is a nilpotent contraction, $T^{n}=0, n \geq 2$, and $\Theta$ is its characteristic function, then there exists $\Omega \in H^{\infty}(B(E))$ such that

$$
\Theta \Omega=\Omega \Theta=z^{n} I_{E}
$$

Therefore, it follows from the inclusion $\Theta \Omega H^{2}(E) \subset \Theta H^{2}(E)$ that

$$
H^{2}(E) \ominus \Theta H^{2}(E) \subset H^{2}(E) \ominus \Theta \Omega H^{2}(E)=H^{2}(E) \ominus z^{n} H^{2}(E)
$$

or

$$
\mathcal{K}_{\Theta} \subset H^{2}(E) \ominus z^{n} H^{2}(E)
$$


Consequently, every $x \in \mathcal{K}_{\Theta}$ has the form

$$
x(z)=\sum_{m=0}^{n-1} \widehat{x}(m) z^{m} .
$$

Then for each $x \in\left(\mathcal{K}_{\Theta}\right)_{1}$ we have

$$
\begin{aligned}
\left\langle M_{\Theta} x, x\right\rangle & =\left\langle P_{\Theta} z x, x\right\rangle \\
& =\left\langle z \sum_{m=0}^{n-1} \widehat{x}(m) z^{m}, \sum_{m=0}^{n-1} \widehat{x}(m) z^{m}\right\rangle \\
& =\left\langle\sum_{m=0}^{n-1} \widehat{x}(m) z^{m+1}, \sum_{m=0}^{n-1} \widehat{x}(m) z^{m}\right\rangle \\
& =\sum_{m=0}^{n-2}\langle\hat{x}(m), \widehat{x}(m+1)\rangle_{E}
\end{aligned}
$$

( $E$ can be identified with a subspace of constant functions in $H^{2}(E)$ ). That is,

$$
\left\langle M_{\Theta} x, x\right\rangle=\sum_{m=0}^{n-2}\langle\widehat{x}(m), \widehat{x}(m+1)\rangle_{E}
$$

Thus, the numerical range $W\left(M_{\Theta}\right)$ of the operator $M_{\Theta}$ is the set

$$
W\left(M_{\Theta}\right)=\left\{\sum_{m=0}^{n-2}\langle\widehat{x}(m), \widehat{x}(m+1)\rangle_{E}: x \in\left(\mathcal{K}_{\Theta}\right)_{1}\right\} .
$$

Proof of the Theorem. We need only to prove the particular case of $\|N\| \leq 1$, and since $\left\|\frac{N}{\|N\|}\right\|=1$, the general result will follow by repeated application of the particular case. Then it is obvious that $W(N)=W\left(M_{\Theta}\right)$ and $w(N)=w\left(M_{\Theta}\right)$, where $M_{\Theta}$ is the model operator of the contraction $N$ and $\Theta$ is its characteristic function. Now we prove that the corresponding set (4) is a circular set. In fact, by virtue of the equality (2) we have that

$$
y_{\zeta}(z) \stackrel{\text { def }}{=} \sum_{m=0}^{n-1} \bar{\zeta}^{m} \widehat{x}(m) z^{m} \in \mathcal{K}_{z^{n} I_{E}}
$$

for each $x=\sum_{m=0}^{n-1} \widehat{x}(m) z^{m} \in \mathcal{K}_{\Theta}$ and $\zeta \in \mathbb{T}$, and therefore, for all $h \in H^{2}(E)$,

$$
0=\left\langle y_{\zeta}, z^{n} h\right\rangle=\left\langle y_{\zeta}, \Omega \Theta h\right\rangle=\left\langle\Omega^{*} y_{\zeta}, \Theta h\right\rangle=\left\langle P_{+} \Omega^{*} y_{\zeta}, \Theta h\right\rangle,
$$

which implies that $\Omega^{*} y_{\zeta} \in L^{2}(E) \ominus \Theta H^{2}(E)$ and $P_{+} \Omega^{*} y_{\zeta} \in \mathcal{K}_{\Theta}$. Since $L^{2}(E) \Theta$ $\Theta H^{2}(E)=\mathcal{K}_{\Theta} \oplus H_{-}^{2}(E)$, we have that $\Omega^{*} y_{\zeta}=P_{+} \Omega^{*} y_{\zeta}+h_{-}$, where $h_{-} \in H_{-}^{2}(E)$. 
Now let the point $0 \neq \lambda \in W\left(M_{\Theta}\right)$ be realized in the element $x=\sum_{m=0}^{n-1} \widehat{x}(m) z^{m} \in$ $\left(\mathcal{K}_{\Theta}\right)_{1}$, i.e., $\lambda=\left\langle M_{\Theta} x, x\right\rangle$. Then, by virtue of (31), for any $t \in[0,2 \pi]$ we have

$$
\begin{aligned}
e^{i t} \lambda & =e^{i t}\left\langle M_{\Theta} x, x\right\rangle=e^{i t} \sum_{m=0}^{n-2}\langle\widehat{x}(m), \widehat{x}(m+1)\rangle_{E} \\
& =\sum_{m=0}^{n-2}\left\langle e^{-i m t} \widehat{x}(m), e^{-i(m+1) t} \widehat{x}(m+1)\right\rangle_{E} \\
& =\sum_{m=0}^{n-2}\left\langle\widehat{y}_{\zeta}(m), \widehat{y}_{\zeta}(m+1)\right\rangle_{E}=\left\langle z y_{\zeta}, y_{\zeta}\right\rangle,
\end{aligned}
$$

where

$$
\begin{gathered}
y_{\zeta}(z)=\sum_{m=0}^{n-1} \widehat{y_{\zeta}}(m) z^{m} \in\left(\mathcal{K}_{z^{n} I_{E}}\right)_{1}, \\
\widehat{y_{\zeta}}(m)=\bar{\zeta}^{m} \widehat{x}(m)=e^{-i m t} \widehat{x}(m) \quad(m \geq 0) .
\end{gathered}
$$

On the other hand,

$$
\begin{aligned}
\left\langle M_{\Theta} P_{+} \Omega^{*} y_{\zeta}, P_{+} \Omega^{*} y_{\zeta}\right\rangle & =\left\langle P_{\Theta} z P_{+} \Omega^{*} y_{\zeta}, P_{+} \Omega^{*} y_{\zeta}\right\rangle=\left\langle z P_{+} \Omega^{*} y_{\zeta}, P_{+} \Omega^{*} y_{\zeta}\right\rangle \\
& =\left\langle z P_{+} \Omega^{*} y_{\zeta}, \Omega^{*} y_{\zeta}\right\rangle=\left\langle z\left(\Omega^{*} y_{\zeta}-h_{-}\right), \Omega^{*} y_{\zeta}\right\rangle \\
& =\left\langle z \Omega^{*} y_{\zeta}, \Omega^{*} y_{\zeta}\right\rangle-\left\langle z h_{-}, \Omega^{*} y_{\zeta}\right\rangle \\
& =\left\langle z y_{\zeta}, y_{\zeta}\right\rangle-\left\langle z h_{-}, \Omega^{*} y_{\zeta}\right\rangle \\
& =e^{i t}\left\langle M_{\Theta} x, x\right\rangle-\left\langle z h_{-}, \Omega^{*} y_{\zeta}\right\rangle=e^{i t} \lambda-\left\langle z h_{-}, \Omega^{*} y_{\zeta}\right\rangle .
\end{aligned}
$$

Thus

or

$$
e^{i t} \lambda=\left\|P_{+} \Omega_{\zeta}^{*} y\right\|^{2}\left\langle M_{\Theta} \frac{P_{+} \Omega^{*} y_{\zeta}}{\left\|P_{+} \Omega^{*} y_{\zeta}\right\|}, \frac{P_{+} \Omega^{*} y_{\zeta}}{\left\|P_{+} \Omega^{*} y_{\zeta}\right\|}\right\rangle+\left\langle z h_{-}, \Omega^{*} y_{\zeta}\right\rangle,
$$

$$
e^{i t} \lambda=\left(1-\left\|h_{-}\right\|^{2}\right)\left\langle M_{\Theta} \frac{P_{+} \Omega^{*} y_{\zeta}}{\left\|P_{+} \Omega^{*} y_{\zeta}\right\|}, \frac{P_{+} \Omega^{*} y_{\zeta}}{\left\|P_{+} \Omega^{*} y_{\zeta}\right\|}\right\rangle+\left\langle z h_{-}, \Omega^{*} y_{\zeta}\right\rangle .
$$

We shall prove that there exists $x_{0} \in\left(\mathcal{K}_{\Theta}\right)_{1}$ such that

$$
\left\langle z h_{-}, \Omega^{*} y_{\zeta}\right\rangle=\left\|h_{-}\right\|^{2}\left\langle M_{\Theta} x_{0}, x_{0}\right\rangle,
$$

and so formulas (5) and (6) will give the desired inclusion $e^{i t} \lambda \in W\left(M_{\Theta}\right)$, since $\left\|h_{-}\right\|^{2}<1$ and $W\left(M_{\Theta}\right)$ is convex.

First recall that if $F_{1}, F_{2}$ are two subspaces of $H$, the angle between $F_{1}$ and $F_{2}$ is the number $\alpha=\alpha_{F_{1}, F_{2}}, 0 \leq \alpha \leq \frac{\pi}{2}$, such that

$$
\cos \alpha=\sup _{x_{1} \in F_{1}, x_{2} \in F_{2}} \frac{\left|\left\langle x_{1}, x_{2}\right\rangle\right|}{\left\|x_{1}\right\|\left\|x_{2}\right\|} .
$$

Since ker $M_{\Theta} \neq\{0\}$, then $0 \in W\left(\left\|h_{-}\right\|^{2} M_{\Theta}\right)$. From the convexity of numerical range it follows that $W\left(\left\|h_{-}\right\|^{2} M_{\Theta}\right)$ contains the line segment $\left[0,\left\|h_{-}\right\|^{2} \lambda\right]$, and moreover, $\overline{\mathbb{D}}_{r} \stackrel{\text { def }}{=}\{z:|z| \leq r\} \subset W\left(\left\|h_{-}\right\|^{2} M_{\Theta}\right)$ for some $r, 0<r<\left\|h_{-}\right\|^{2}|\lambda|$. Let $L_{1} \subset L^{2}(E)$ and $L_{2} \subset L^{2}(E)$ be the subspaces such that $z h_{-} \in L_{1}$ and $\Omega^{*} y_{\zeta} \in L_{2}$. Recall that two contractive functions $\Theta_{1} \in H^{\infty}\left(B\left(E_{1}, E_{2}\right)\right), \Theta_{2} \in$ 
$H^{\infty}\left(B\left(E_{1}^{\prime}, E_{2}^{\prime}\right)\right)$ coincide (see [SF], Chapter 5 ) if there exist unitary operators $\tau_{1}: E_{1} \rightarrow E_{1}^{\prime}$ and $\tau_{2}: E_{2} \rightarrow E_{2}^{\prime}$ such that $\Theta_{2}(z)=\tau_{2} \Theta_{1}(z) \tau_{1}^{-1},|z|<1$. It is well known that two completely nonunitary contractions are unitarily equivalent if and only if their characteristic functions coincide. Therefore, we can assume the function $\Omega$ and the subspaces $L_{1}, L_{2}$ are such that $\left\|h_{-}\right\| \cos \alpha_{L_{1}, L_{2}} \leq r$. Then we have

$$
\begin{aligned}
\left|\left\langle z h_{-}, \Omega^{*} y_{\zeta}\right\rangle\right| & =\left\|z h_{-}\right\| \frac{\left|\left\langle z h_{-}, \Omega^{*} y_{\zeta}\right\rangle\right|}{\left\|z h_{-}\right\|\left\|\Omega^{*} y_{\zeta}\right\|} \\
& \leq\left\|h_{-}\right\| \sup _{x_{1} \in L_{1}, x_{2} \in L_{2}} \frac{\left|\left\langle x_{1}, x_{2}\right\rangle\right|}{\left\|x_{1}\right\|\left\|x_{2}\right\|} \\
& =\left\|h_{-}\right\| \cos \alpha_{L_{1}, L_{2}} \leq r,
\end{aligned}
$$

which shows that $\left\langle z h_{-}, \Omega^{*} y_{\zeta}\right\rangle \in \overline{\mathbb{D}}_{r}$. Since $\overline{\mathbb{D}}_{r} \subset W\left(\left\|h_{-}\right\|^{2} M_{\Theta}\right)$, there exists $x_{0} \in\left(\mathcal{K}_{\Theta}\right)_{1}$ such that

$$
\left\langle z h_{-}, \Omega^{*} y_{\zeta}\right\rangle=\left\langle\left\|h_{-}\right\|^{2} M_{\Theta} x_{0}, x_{0}\right\rangle=\left\|h_{-}\right\|^{2}\left\langle M_{\Theta} x_{0}, x_{0}\right\rangle .
$$

Consequently, $e^{i t} \lambda \in W\left(M_{\Theta}\right)$ for each $t \in[0,2 \pi]$, which means that $W\left(M_{\Theta}\right)$ is a circular set. On the other hand, since the numerical range is convex, $W\left(M_{\Theta}\right)$ is a circle (open or closed) with center at zero, which completes the proof.

Corollary. If $N \in B(H)$ is a compact nilpotent operator, then $W(N)$ is a closed disk centered at 0 and with radius not greater than $\|N\| \cos \frac{\pi}{n+1}$.

Proof. It follows from the result of Lancaster $[\mathrm{L}]$ that if $\mathcal{K} \in B(H)$ is compact, then $W(\mathcal{K})$ is closed if and only if $0 \in W(\mathcal{K})$. It remains only to apply the theorem.

Example 1. Let $\left(V_{0} f\right)(x)=\int_{-x}^{x} f(t) d t$ be a skew-symmetric Volterra operator acting on the space $L^{2}(-1,1)$. Then its numerical range $W\left(V_{0}\right)$ is a closed circle with center at 0 and radius $\frac{2}{\pi}$, i.e., $W\left(V_{0}\right)=\overline{\mathbb{D}}_{\frac{2}{\pi}}$.

Proof. Observe that the set of values of the operator $V_{0}$ is contained in the set of all odd functions from the space $L^{2}(-1,1)$ and for the odd function $f, V_{0} f=0$. This means that $V_{0}^{2}=0$, i.e., $V_{0}$ is a nilpotent operator with the power of nilpotency 2 . Then by our theorem, $W\left(V_{0}\right)$ is a circle with center at zero. Since $V_{0}$ is a compact operator and $0 \in W\left(V_{0}\right)$, by the corollary, $W\left(V_{0}\right)$ is a closed set. So, $W\left(V_{0}\right)$ is a closed circle. Now we calculate its radius. By inequality (1),$w\left(V_{0}\right) \leq \frac{\left\|V_{0}\right\|}{2}$. On the other hand, $w\left(V_{0}\right) \geq \frac{\left\|V_{0}\right\|}{2}$, and hence $w\left(V_{0}\right)=\frac{\left\|V_{0}\right\|}{2}$. Since $\left\|V_{0}\right\|=\frac{4}{\pi}$ (see []] ), we have that $w\left(V_{0}\right)=\frac{2}{\pi}$. This completes the proof.

The following example is easy to verify.

Example 2. Consider the diagonal operator $D=\operatorname{diag}\left\{\frac{1}{2}, \frac{2}{3}, \frac{3}{4}, \ldots\right\}$ and $N=$ $\left[\begin{array}{ll}0 & D \\ 0 & 0\end{array}\right]$ acting in $\ell^{2}$. Then $N$ is not compact, $N^{2}=0$, and $W(N)$ is the open disk centered at 0 with radius $\frac{1}{2}$, i.e., $W(N)=\mathbb{D}_{\frac{1}{2}}$. 
Remark. Recall that the $k$-numerical range $W_{k}(A)$ of the operator $A \in B(H)$ is defined by

$$
W_{k}(A)=\left\{\lambda \in \mathbb{C}: \lambda=\sum_{i=1}^{k}\left\langle A x_{i}, x_{i}\right\rangle,\right.
$$

where $\left\{x_{1}, \ldots, x_{k}\right\}$ are $k$ orthonormal vectors in $\left.H\right\}$.

When $k=1$, this reduces to the classical numerical range $W(A)$. The most fundamental property of the $k$-numerical range is its convexity (see $[\mathrm{H}]$ ). Using this property and arguments similar to those in the proof of the theorem, we can also obtain the analogous characterization for the $k$-numerical range $W_{k}(N)$ of a nilpotent operator $N \in B(H)$, which is also contained among other general results of the paper $[\mathrm{LT}]$.

I am grateful to the referee for his constructive suggestions and for pointing out the reference $[\mathrm{LT}$.

\section{REFERENCES}

[GR] K. E. Gustafson and D. K. M. Rao, Numerical range. The field of values of linear operators and matrices, Springer-Verlag, New York, 1997. MR 98b:47008

[HH] U. Haagerup and P. de la Harpe, The numerical radius of a nilpotent operator on a Hilbert space, Proc. Amer. Math. Soc. 115 (1992), 371-379. MR 92i:47002

[H] P. R. Halmos, A Hilbert space problem book, 2nd ed., Graduate Texts in Mathematics, no. 19, Springer-Verlag, New York, 1982. MR 84e:47001

[L] J. S. Lancaster, The boundary of the numerical range, Proc. Amer. Math. Soc. 49 (1975), 393-398. MR 51:8851

[LT] C. K. Li and N. K. Tsing, Matrices with circular symmetry on their unitary orbits and C-numerical ranges, Proc. Amer. Math. Soc. 111 (1991), 19-28. MR 91d:15054

[N] N. K. Nikolski, Treatise on the shift operator, Springer-Verlag, Heidelberg, 1986.

[SF] B. Sz.-Nagy and C. Foias, Harmonic analysis of operators on Hilbert space, North-Holland, Amsterdam, 1970. MR 43:947

Institute For Mathematics And Mechanics, Azerbaijanian National Academy of Sciences, F.Agaev, 9, 370141 Baku, Azerbaijan

Current address: Department of Mathematics, Faculty of Arts and Sciences, Suleyman Demirel University, 32260 Isparta, Turkey

E-mail address: garayev@fef.sdu.edu.tr 